10 - ORIGINAL ARTICLES

\title{
Key Points for Curbing Cardiopulmonary Bypass Inflammation ${ }^{1}$
}

\author{
Paulo Roberto Barbosa Evora ${ }^{\mathrm{I}}$, Camila Bottura ${ }^{\mathrm{II}}$, Livia Arcêncio" ${ }^{\mathrm{II}}$, Agnes Afrodite Sumarelli Albuquerque ${ }^{\mathrm{IV}}$, Patrícia Martinez \\ Évorav $^{v}$ Alfredo José Rodrigues ${ }^{\mathrm{VI}}$
}

DOI: $h t t p: / / d x$. doi.org/10.1590/S0102-86502016001300010

\begin{abstract}
IFull Professor, Department of Surgery and Anatomy, Ribeirão Preto Medical School, University of São Paulo (FMRP-USP). Intellectual and scientific content of the study, supervised all phases, manuscript writing.

IIFellow Master degree, Program in Medical Surgical Clinic from the Department of Surgery and Anatomy, Ribeirão Preto Medical School, University of São Paulo (FMRP-USP). Acquisition and interpretation of data, collection of study information, manuscript writing.

IIIFellow Master degree, Program in Medical Surgical Clinic from the Department of Surgery and Anatomy, Ribeirão Preto Medical School, University of São Paulo (FMRP-USP). Acquisition and interpretation of data, collection of study information, manuscript writing.

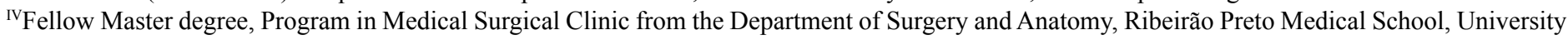
of São Paulo (FMRP-USP). Acquisition, collection of study information.

vFellow Master degree, Program IN Veterinary Pathology Department of Animal Pathology, Veterinary School, São Paulo State University (UNESP), Campus of Jaboticabal, Brazil. Acquisition, collection of study information, manuscript writing.

${ }^{\mathrm{VI}} \mathrm{MD}, \mathrm{PhD}$, Head of Postgraduate Program in Medical Surgical Clinic from the Department of Surgery and Anatomy, Ribeirão Preto Medical School, University of São Paulo (FMRP-USP). Acquisition and interpretation of data, collection of study information.
\end{abstract}

\section{ABSTRACT}

PURPOSE: Cardiopulmonary bypass (CPB) procedures are thought to activate systemic inflammatory reaction syndrome (SIRS). Strategies to curb systemic inflammation have been previously described. However, none of them is adequate, since "curbing" the extent of the inflammatory response requires a multimodal approach. The aim of the present mini-review is to discuss the main key points about the main principles in cardiopulmonary bypass curbing inflammation.

METHODS: No systematic literature search (MEDLINE) and extracted data from the accumulated experience of the authors. The preconceived idea of an association between severe inflammation and coagulation disorders is reviewed. Also, some fundamental concepts, CPB inflammatory biomarkers, the vasoplegic syndrome and the need forindividual CPB protocols for children, diabetes and old patients, are discussed.

CONCLUSION: The ways in which surgical technique (atraumatic vein harvest, biocompatibility and shear resistance of the circuit, monitoring, minimizing organ ischemia, minimal cross-clamping trauma, and blood management) are thought to curb SIRS induced by $\mathrm{CPB}$ and affect positively the patient outcome.Improved patient outcomes are strongly associated with these modalities of care, more than single or combinatorial drug strategies (aprotinin, tranexamic acid, pentoxifylline) or CPB modalities (minicircuits, heparin-coated circuits, retrograde autologous prime).

Key words: Cardiopulmonary bypass. Cardiac surgery. Inflammation. Systemic inflammatory reaction syndrome. SIRS. 


\section{Introduction}

The contact of blood with a non-physiological surface during cardiopulmonary bypass (CPB) surgery in thought to induce a systemic inflammatory reaction syndrome (SIRS). CPB plastic components and the CPB procedures, per se, are thought to trigger the inflammatory response that results in the subsequent myocardial injury. Today, this paradigm is strongly debated, mainly because an inflammatory response is still present in patients undergoing off-pump surgery. In addition, O'Brien et al. (2011) reported that transcatheter aortic valve implantation (TAVI) (which reduces surgical trauma and avoids the need for CPB) does not attenuate the patients' innate inflammatory response ${ }^{1}$.

Many studies have shown that blood contact with the operation wound may handle the inflammatory reaction. This hypothesis proposes that blood coming into contact with serous membranes (pleura and pericardium) causes fibrinolytic activity and increased bleeding, which agrees with recent advances in our knowledge and understanding about the association between coagulation and inflammation. As CPB coagulation control is one of the most serious problems, it is possible that inadequate heparin use could handle inappropriate, imperceptible anticoagulation and therefore also for triggering inflammation ${ }^{2}$.

Minimizing systemic inflammation (SIRS) as a primary strategy for inhibiting the inflammatory response has been described. None of these strategies is adequate, but "curbing" the extent of the inflammatory response requires a multimodal approach.

Finally, since CPB techniques are clearly divided into protocols for adults and children, elderly and diabetic patients might merit more appropriate protocols since these populations has atypical immunologic characteristics ${ }^{2}$. The review did not adopt any specific method of the literature search. It was taking into account the MEDLINE database and previous authors publications.

\section{Brief physiopathology of the CPB inflammatory} reaction

The physiopathology of the CPB systemic inflammatory response is multifactorial, with no final consensus about its actual mechanism. It canbe divided into two main phases: "early" and "late". The first phase occurs as a result of blood contact with non endothelial surfaces ("contact activation"). The late phase is caused by ischemia-reperfusion injury (I/R injury), endotoxemia, coagulation disorders, and heparin/protamine reactions ${ }^{3,4}$. Overall, the early phase is caused by 1) cellular components (endothelial cells, neutrophils, monocytes, lymphocytes, and platelets), and; 2) humoral components (contact, intrinsic coagulation, extrinsic coagulation, complement, and fibrinolysis) $)^{3,4}$. The late phase is related to $I / R$ injury andendotoxemia. $I / R$ injury has two physiopathological components: 1) leucocyte-dependent (neutrophil-endothelial interaction) and 2) leucocyte-independent (Reactive oxygen species, arachidonic acid metabolites, and cytokine release). Endotoxemia implies complement activation, cytokine release, nitric oxide (NO) release, and increased oxygen consumption. More recently, it was observed that this antiinflammatory response might be initiated during and after CPB.

To define the magnitude of the CPB systemic inflammatory response, Landis (2007) suggests a kind of "never ending story" withrare yet defined endpoints, such as myocardial injury, stroke, and renal failure requiring dialysis ${ }^{5}$. The vast majority of studies wrongly suggest that the problem is confined to inflammation. In fact, it is characterized by a systemic imbalance of natural defenses against injury (coagulation, fibrinolysis, complement activation, oxidative stress, and immune cell activation). Therefore, a better definition would be to think in terms of a "systemic host response."

The mechanisms involved are presented in Table $1^{2}$. Also, the factors influencing the CPB systemic inflammatory response are shown in Table $2^{6}$.

TABLE 1 - $\quad$ Individual inflammatory Cardiopulmonary bypass (CPB) mechanisms (adapted from [2]. RevBrasCirCardiovasc23(1):78-92, 2008)

1. The clinical relevance of complement activation is still uncertain. Several studies have linked postoperative morbidity to complement activation.

2. Preventing neutrophil adhesion may carry practical benefits, but these benefits may be associated with a greater risk of infection.

3. Leukocytes activation may release large amounts of oxygen free radicals, including superoxide anion, hydrogen peroxide, hydroxyl radical and oxygen itself. These radicals can increase the permeability of lipid membranes that can affect cardiac and pulmonary function.

4. The metabolic by-products of arachidonic acid (prostaglandins, leukotrienes and thromboxane A2) may be counterbalanced by the concomitant production of vasodilatory prostaglandins such as prostacyclin (PGI2). Leukotrienes may be responsible for the increase in capillary permeability.

5. Endotoxin levels may increase during and after CPB. The sources of endotoxins are highly diverse. However, the most significant source is the intestines. Splanchnic vasoconstriction during CPB may induce ischemia and increase of the permeability of intestinal loops resulting with the release of endotoxins into the blood flow. The endotoxins levels are associated with initial vasoconstriction, the time of aortic clamping and the hypo-oncotic state during CPB. 
6 . The release of cytokines may be stimulated by a number of factors, including ischemia-reperfusion, complement activation, the release of endotoxins and the effect of other cytokines.

7. Platelet-activating factor (PAF) plays a crucial role in the lesions of myocardial ischemia-reperfusion and may also have hemodynamic deleterious effects during $\mathrm{CPB}$.

8 . The excessive production of induced nitric oxide synthase (iNOS) may result from these multiple mechanisms, being the biggest cause of the vasoplegic syndrome.

9. There may be an increase in endothelin levels during CPB, surgical treatment of congenital cardiopathy, valve disease and coronary artery by-pass graft (CABG). Its role is not clear in the physiopathology of post-CPB vasoplegia. If vasoplegia occurs as a consequence of a massive release of $\mathrm{NO}$, then the

vasoconstricting effect of endothelin is not apparent.

10. The incidence of inflammation occuring during the $\mathrm{CPB}$ circuit is higher with the use of bubble oxygenators than with membrane oxygenators. The physiopathology of this remains nuclear.

TABLE 2 - Factors influencing the Cardiopulmonary bypass (CPB) systemic inflammatory response (Adapted from [6] Asian Cardiovasc Thorac Ann 2005; 13: 382-395, 2005.)

\section{Factors Influencing Systemic Inflammatory Response}

1. Biomaterial-Dependent Factors: extracorporeal circuit, oxygenator and pump.

2. Biomaterial-Independent Factors: extracorporeal perfusion factors (priming solutions, cardioplegia, pulsatile or non-pulsatile perfusion, temperature during $\mathrm{CPB}$.

3. Preoperative Factors: morbid conditions.

4. Perioperative Hemodynamic Factors: low cardiac output, splanchnic hypoperfusion, anesthesia (technique, anesthetic agentsand drugs).

5. Lung Management during CPB.

6. Surgical Factors: incision and approach, duration, cardiotomy blood management, shear stress, transfusion.

7. Postoperative factors: continuous renal replacement therapies, mechanical ventilation.

CPB during pediatric cardiac surgery often elicits a systemic inflammatory response followed by a compromised immune response, which has been attributed to the morbidity of postoperative infection. However, the underlying mechanism (s) have not yet been fully elucidated ${ }^{7}$. Li et al. (2014) hypothesized that CPB inhibits the activation of the Toll-like receptor (TLR) signal transduction pathways, resulting in an immunosuppressive state in their study of 20 children undergoing pediatric cardiac surgery. These authors demonstrated that $\mathrm{CPB}$ differentially affected lipopolysaccharide (LPS) or bacterial lipoprotein (BLP)-stimulated ex vivo production of proinflammatory and anti-inflammatory cytokines, with significantly diminished tumor necrosis factor $\alpha$, interleukin (IL) $1 \beta$, IL-6, and IL-8, but substantially enhanced IL-10 production. These results indicated that CPB during pediatric cardiac surgery results in a significantly reduced production of inflammatory cytokines, which is associated with reduced inflammatory response after CPB in children with congenital heart disease ${ }^{7}$. In fact, while inflammation is frequent following pediatric $\mathrm{CPB}$, serious consequences only affect a small number of patients ${ }^{8,9}$. Also, many studies were performed in small groups of patients and were, therefore, likely to beunderpowered ${ }^{8,10}$.

It is well known that oxidative stress is about two times higher in diabetic patients that undergoing on-pump heart surgery develop more severe complications. Furthermore, some studies have reported that the inflammatory response is different in patients with diabetes when compared with non-diabetic patients. Onorati et al. (2011) evaluated the endothelial/cytokine response (ECR) after cardiac surgery and its role in infective/inflammatory complications of type II diabetic patients ${ }^{11}$. The perioperative ECR of 60 diabetic patients undergoing CPB (Group A) was compared to that of 60 non-diabetic patients (Group B). Hemodynamics, endothelial markers [vascular endothelial growth factor (VEGF) and monocyte chemotactic protein-1 (MCP-1)], pro-inflammatory (IL-2, IL-6, IL-8), and anti-inflammatory cytokines (IL-10) were analyzed preoperatively ( $\mathrm{T} 0$ ), at time of aortic declamping (T1), at intensive care unit admission (T2), at $12 \mathrm{~h}$ (T3) and 24 h (T4) postoperatively. Postoperative infective/inflammatory complications were registered, and the related ECR analyzed. The hemodynamics of these patients were comparable. No differences were found in perioperative IL- 6 and IL- 8 between the two groups. However, the diabetic patients had significantly higher endothelial activation (VEGF) with lower IL-10 and lower IL-2 secretion. Infections developed in $23.3 \%$, and inflammatory complications in $13.3 \%$ of the patients with diabetes. Those developing infections showed significantly lower IL-2 than patients without infections, whereas those with complicated inflammatory lung or renal injury had higher MCP-1leakage with lower IL-10. The researchers concluded that the diabetic patients showed greater endothelial activation and lower anti-inflammatory responses to CPB than non-diabetic patients. Infections in diabetic patients correlated with suppressed IL-2 while inflammatory complications correlated to higher endothelial activation and less anti-inflammatory cytokine secretion ${ }^{11}$.

These observations warrant further attention as a particular characteristic of the inflammatory reaction of children and diabetic patients and, maybe, elderly patients under CPB. 


\section{Biomarkers}

Descriptive studies are measuring various clinical and biochemical parameters in patients undergoing off-pump coronary artery bypass graft (CABG) surgery and compare them with those of patients undergoing conventional cardiac surgery. In this way, Asimakopoulos $(2001)^{12}$ proposed that 1) CPB appear to be responsible for the activation of complement that is known to occur during cardiac surgery; 2) CPB specifically promotes secretion of the proinflammatory cytokines interleukin-8 (IL-8) and tumor necrosis factor- $\alpha(\mathrm{TNF}-\alpha)$, and the anti-inflammatory cytokine IL-10; 3) Plasma levels of C-reactive protein (CRP) and IL-6 are raised equally in both patient groups. IL-6 levels, however, were reduced in minimally invasive direct coronary artery bypass (MIDCAB) patients compared to that of off-pump coronary artery bypass (OPCAB) patients in one study. These data suggest that operative trauma, rather than $\mathrm{CPB}$ per se, initiates the release of IL-6. 4) CPB promotes activation of neutrophils as measured by plasma elastase levels ${ }^{10,11}$ and bactericidal permeability increasing protein $(\mathrm{BPI})^{12}$.

There is strong evidence that NO has a central role in the progressive vasodilatation associated with various forms of circulatory shock in patients undergoing cardiac surgeries for CPB (CPB), including systemic inflammatory response syndrome (SIRS). If CPB procedures, per se, are the inciting stimuli for inflammation, plasma nitrate/nitrite (NOx) excretion would be expected to be higher in these patients compared to patients undergoing surgery without $\mathrm{CPB}^{13}$. Besides $\mathrm{CRP}$, nowadays, routine nitrite/nitrate (NOx) is an easy and inexpensive method. Therefore, we hypothesized that increased levels of NOx would be predictive of the vasoplegic syndrome. Thirty patients were assigned to three groups: Group 1-CABG roller pump CPB; Group 2-CABG centrifugal vortex pump CPB; and Group $3-$ heart valve surgery roller pump CPB. Venous blood samples for the detection chemiluminescence plasma NOx levels were taken at the following time points: (1) before anesthesia induction; (2) after anesthesia induction; (3) before heparin infusion; (4) after heparin infusion; (5) CPB-30 minutes; (6) CPB-60 minutes; (7) before protamine (8) after protamine infusion, and (9) on return to the recovery area. There were no intergroup differences in age and anesthetic regimen, and the number of arteries grafted was not different between the CABG groups. There were no statistical differences in NOx levels among the three groups of patients or the surgery time. Also, there was no correlation between NOx, lactate, and hemoglobin levels. Therefore, plasma NOx is not useful as a biomarker of the onset of an inflammatory response, which may or may not lead to SIRS and vasoplegic syndrome, which was unexpected ${ }^{13}$. (Figure 1)

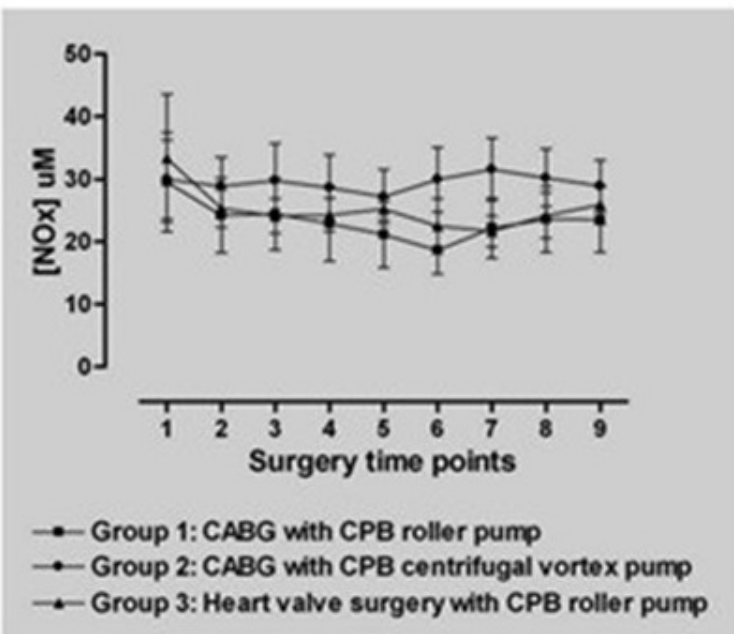

FIGURE 1 - Plasma nitrate/nitrite (NOx) in patients undergoing cardiac surgery. Surgery time plasma samples points: 1) Before anesthesia induction; 2) After anesthesia induction; 3) Before heparin infusion; 4) After heparin infusion; 5) CPB-30 minutes; 6) CPB 60 minutes; 7) Before protamine infusion; 8) After protamine infusion and; 9) On return to the recovery area. $(n=30,10$ patients per group, ANOVA two-way, differences not significant $\mathrm{p}<0.05$ ) (Viaro et al, J Card Surg 23: 336-338, 2008)

The CPB and cardiac surgery inflammatory response is measurable by an increase in the concentration of CRP, a nonspecific inflammatory marker. Previous publications have demonstrated typical perioperative CRP concentration profiles in cases of uncomplicated aortic valve replacement (AVR) with CPB. A regression analysis of modifying factors showed that chronic disease (heart failure, diabetes, and pulmonary disease), along with obesity and sex, all tend to influence the CRP response ${ }^{1}$. Even though it is a non-specific inflammatory marker, CRP is measured routinely by hospital laboratories and would, therefore, be a useful tool in cardiac surgery.

When considering $\mathrm{CPB}$ inflammatory reactions, it is important to understand that the balance between pro and antiinflammatory agents provides more valuable information than the levels of pro-inflammatory biomarkers alone, and further studies should consider this.

A useful fact is that alkaline phosphatase activity decreases after cardiothoracic surgery in infants. Low postoperative alkaline phosphatase activity is independently associated with increased procalcitonin, increased vasoactive/inotropic support, prolonged intubation time, and prolonged hospital stay. Alkaline phosphatase may serve as a biomarker and potential modulator of post-operative support and inflammation following cardiothoracic surgery in infants ${ }^{14}$. These observations, taking into account the binomial cost-benefit, warrant further study since, as with CRP, alkaline phosphatase is measured routinely by hospital laboratories and would, therefore, be a useful tool in cardiac surgery. 
It has been reported that $\mathrm{CPB}$ induces greater oxidative stress in patients with diabetes than in those without diabetes and that the inflammatory reaction is qualitatively different between these two groups of patients. The neutrophil chemotactic cytokine interleukin-8 (IL8) was found to be significantly more increased after CPB in diabetic patients than in non diabetic patients ${ }^{15}$.

Gene expression profiling after CPB is quantitatively and qualitatively different in patients with diabetes. These results have significant implications for the design of tailored myocardial protection and operative strategies for diabetic patients undergoing $\mathrm{CPB} / \mathrm{C}$. However, gene expression profiling is still not useful for current cardiac surgery day-by-day practice.

\section{Curbing cardiopulmonary bypass inflammation}

Aside from the mechanical and pharmacological approaches to curbing CPB inflammation, CPB quality (materials, laboratory control, well-trained per fusionists, etc.) is paramount. Surgeons must maintain and improve their understanding of CPB, by retaining educational leadership over their team. On the other hand, there is individual tolerance to the CPB apparatus because the quality of the material ensures a significant margin of safety for heart operations.

According to Durandy (2014), one of the greatest challenges is how to modulate the systemic inflammatory response so that excessive inflammation in controlled while preserving a level of inflammation needed for host defense and wound healing ${ }^{8}$. Further research is required to substantiate this.

Taking into account pharmacologic strategies for curbing the CPB inflammatory response, Landis (2007), published an overview on the subject, clearly stating that a single "magic bullet" is implausible, because the phenomenon is a multifaceted defensive reaction ${ }^{5}$. Therefore, the best chance lies with combinatorial-or promiscuous-pharmacotherapy. Combinations of antifibrinolytics, anti-coagulants targeted higher up the coagulation cascade, anti-thrombin receptor therapy, improved coated circuits, anticomplement, anti-leukocyte, and antioxidant therapies may suppress sufficient arms of SIRS to be clinically useful.

Table 3 presents the non-pharmacologic techniques and technology that potentially curb the CPB inflammation reaction. The Table 4 lists the vast majority of potential pharmacologically useful agents. Raja and Dreyfus (2005) superbly describe all these curbing alternatives, which is a recommended reader ${ }^{6}$.

TABLE 3 - Non-pharmacologic techniques and technology with the potential capacity to curb the cardiopulmonary bypass (CPB) induced an inflammatory reaction. \# Font: MEDLINE SEARCH (cardiac surgery AND cardiopulmonary bypass AND inflammation AND ("non-pharmacologic approach")

A - NOVEL CARDIAC SURGICAL TECHNIQUE AND TECHNOLOGY

Minimized extracorporeal circulation system

Off-pump coronary bypass

Minimally invasive cardiac surgery

Transcatheter aortic valve implantation (TAVI)
PUBLICATIONS

(N)

08

111

15

02
YEAR INTERVAL

$1999-2010$

$1997-2014$

$1998-2013$

2011

\section{C - FILTRATION TECHNIQUES}

Hemofiltration 
TABLE 4 - Pharmacologic strategies to curb the cardiopulmonary bypass (CPB) induced a systemic inflammatory reaction. \# Font: MEDLINE (cardiac surgery AND cardiopulmonary bypass AND inflammation AND ("drug")

\begin{tabular}{|c|c|c|}
\hline $\begin{array}{l}\text { HARMACOLOGIC } \\
\text { STRATEGIES }\end{array}$ & $\begin{array}{l}\text { PUBLICATIONS } \\
(\mathrm{N})\end{array}$ & $\begin{array}{c}\text { YEAR } \\
\text { INTERVAL }\end{array}$ \\
\hline Aprotinin & 60 & $1995-2013$ \\
\hline Pentoxyfilline & 06 & $1993-2006$ \\
\hline $\begin{array}{c}\text { Free-radical scavengers } \\
\text { and antioxidants }\end{array}$ & 63 & $1985-2013$ \\
\hline $\begin{array}{l}\text { High doses of Vitamin C } \\
\text { and Vitamin E }\end{array}$ & 62 & $2005-2012$ \\
\hline Allopurinol & 01 & 2012 \\
\hline Manitol & 03 & $2006-2013$ \\
\hline $\begin{array}{l}\text { Phosphodiesterase } \\
\text { inhibitors (sildenafil) }\end{array}$ & 00 & - \\
\hline $\mathrm{N}$-acetylcysteine & 05 & $2004-2009$ \\
\hline Corticosteroids & 76 & $1989-2014$ \\
\hline Statins & 17 & $2005-2014$ \\
\hline $\begin{array}{c}\text { Cyclooxygenase } \\
\text { inhibitors (milrinone) }\end{array}$ & 04 & $1999-2013$ \\
\hline $\begin{array}{c}\text { Complemente-directed } \\
\text { therapies }\end{array}$ & 98 & $1988-2014$ \\
\hline
\end{tabular}

The current literature on worldwide cardiac surgery excellence supports the overestimation of pathological inflammation in human CPB surgery. However, when it does occur it is catastrophic and is associated with high morbidity and mortality.

The ways in which surgical technique (atraumatic vein harvest, biocompatibility, and shear resistance within the circuit, monitoring, minimizing organ ischemia, minimal cross-clamping trauma, and blood management) are thought to affect patient outcome. Improved patient outcomes are strongly associated with these modalities of care, more than single or combinatorial drug strategies.

Currently, this may represent the CPB inflammationcurbing paradigm. We speculate that pathological inflammation in the human CPB surgery is overestimated. However, when it occurs, it is catastrophic and associated with high morbidity and mortality.

\section{CPB vasoplegic syndrome}

The modern era of heart surgery began when the CPB technique was introduced in the early 1950s. CPB is essential for the majority of cardiac surgery, but an undesirable inflammatory reaction occurs because of its use. To understand the vasoplegic syndrome physiopathology there are many hypotheses including a) viral and bacterial infections, b) immunological reactions related to anti myocardial antibodies, c) anaphylactoid reactions associated with anesthetics, protamine, heparin, and the $\mathrm{CPB}$ circuit itself ${ }^{16}$. Many factors during CPB, both dependent on (exposure of blood to surfaces and not physiological conditions), or independent of the CPB material (surgical trauma, ischemia-reperfusion of the organs, changes in body temperature, and release of endotoxins), have been reported as inducers of a complex inflammatory response.

Gomes et al. described the vasoplegic syndrome associated with $\mathrm{CPB}^{17}$ in 1994 and Evora et al. proposed that vasoplegia be dependent on the cyclic GMP system ${ }^{18,19}$. Since it is clinically impossible to block the production of NO from L-arginine during surgery, it was proposed instead to inhibit guanylate cyclase by the use of methylene blue (MB). Nowadays, this approach seems to be the most reasonable since it does not interfere with NO synthesis, and it is a medication widely used in other clinical conditions. The action of MB involves inhibition of guanylate cyclase, thus avoiding the increase of cyclic GMP and avoiding the endothelium-dependent relaxation mediated by $\mathrm{NO}^{2}$.

Although MB has been used to treat vasoplegic syndrome for over 20 years, few human studies can approve a treatment protocol. We include only three studies in this CPB review, which reported substantial data in studies involving larger numbers of patients $^{20}$. In 2003, Leyh et al. ${ }^{21}$ reported 54 cases of cardiac surgery patients with no bacterial endocarditis who were treated with $\mathrm{MB}$ and where a response to the treatment was achieved in over $90 \%$ of patients. Levin et al. ${ }^{21}$ reported an incidence of $8.8 \%$ vasoplegic syndrome in a study of 638 patients. In this study, the 56 vasoplegic patients were randomized to receive either MB or placebo.

Regarding the prevention of the vasoplegic syndrome, Ozal et al..$^{22}$, in a prospective randomized study, showed that MB was associated with a lower incidence of vasoplegia and less use of sympathetic amines. The criterion for inclusion of more patients in the study protocol was the use of heparin and angiotensinconverting-enzyme (ACE) inhibitors. These two drugs are, up to now, the only ones considered as a risk factor for the vasoplegic syndrome.

Emergency cardiac valve surgery due to acute endocarditis has a poor outcome in many patients because of the huge inflammatory reaction. An early example of such a patient was a drug-addicted young man with native aortic valve endocarditis. The patient received a bileaflet valve prosthesis ( $\mathrm{St}$ 
Jude Medical, Inc., St Paul, Minn). A high dose of norepinephrine was necessary to maintain reasonable blood pressure during CPB. After CPB weaning, he was hypotensive and had a high cardiac output, low systemic vascular resistance, and pulmonary edema. The arterial oxygen saturation was below $80 \%$, even though he was being ventilated with $100 \%$ oxygen and positive end-expiratory pressure. He was prescribed $\mathrm{MB}$ in a continuous infusion in followed by a bolus of $3 \mathrm{mg} / \mathrm{kg}$ (in $100 \mathrm{~mL}$ of $5 \%$ glucose in water) twice a day. Even though the mean arterial pressure did not increase, and even with norepinephrine, the cardiac output gradually decreased, and the systemic vascular resistance increased. Also, the rapid resolution of lung edema resulting in higher arterial oxygen saturation was remarkable. After this pilot case, a protocol was adopted beginning MB infusion as soon the hemodynamic recorder shows low systemic vascular resistance index. If the hypotension persists irresponsive to amines and high CPB flow, MB infusion should be repeated on pump. Therefore, the NO/cGMP pathway blockage is a lifesaving tool for curbing the CPB inflammatory reaction ${ }^{23-25}$.

\section{Key points}

- Based on, the literature overview and the worldwide cardiac surgery excellence, it seems possible that pathological inflammation during human CPB surgery is overestimated. However, when it does occur it is catastrophic and is associated with high morbidity and mortality.

- The ways in which surgical technique (atraumatic vein harvest, biocompatibility, and shear resistance within the circuit, monitoring, minimizing organ ischemia, minimal cross-clamping trauma, and blood management) are thought to affect patient outcome. Improved patient outcomes are strongly associated with these modalities of care, more than single or combinatorial drug strategies.

- The physiopathology of the CPB systemic inflammatory response is multifactorial, with no final consensus about its actual mechanism. It can be divided into two main phases: "early" and "late." The early phase occurs as a result of blood contact with non-endothelial surfaces ("contact activation"), and the late phase is driven by ischemia-reperfusion injury ( $\mathrm{I} / \mathrm{R}$ injury), endotoxemia, coagulation disorders, and reactions to heparin/protamine.
- While inflammation persists following pediatric $\mathrm{CPB}$, serious consequences only affect a small number of pediatric patients. Many of these studies, however, were performed with small groups of patients and were likely to be underpowered in terms of making reliable evidencebased conclusions.

- Diabetic patients showed higher endothelial activation and lower anti-inflammatory responses to $\mathrm{CPB}$ than nondiabetic patients. Infections in diabetic patients correlated with depressed IL-2 while inflammatory complications correlated to higher endothelial activation and lower anti-inflammatory cytokine secretion.

- Chronic disease (heart failure, diabetes, and pulmonary disease), along with obesity and sex, all tend to influence the CRP response.

- Despite being an unspecific marker of inflammation, Creactive protein (CRP) is measured routinely by hospital laboratories and would, therefore, make a useful marker in cardiac surgery.

- The balance between pro and anti-inflammatory agents provides more valuable information than the levels of pro-inflammatory biomarkers alone.

- Like CRP, alkaline phosphatase is measured routinely in hospital laboratories, and could, therefore, be a useful marker in cardiac surgery.

- It has been reported that $\mathrm{CPB}$ induces greater oxidative stress in patients with diabetes than in those without diabetes and that the inflammatory reaction is qualitatively different in these two groups of patients.

- One of the greatest challenges is how to modulate the systemic inflammatory response to control excessive inflammation, but while preserving a level of inflammation required for host defense and wound healing. These observations should be the focus of further research.

- There are two core methods with the potential for preventing or reducing CPB inflammation: 1) non-pharmacologic techniques and technology, and 2) pharmacologic agents.

- The most studied drugs are corticosteroids and aprotinin. The NO/cGMP pathway blockage by MB could be a lifesaving tool for curbing the $\mathrm{CPB}$ inflammatory reaction. 


\section{References}

1. O’Brien B, Pasic M, Kuppe H, Hetzer R, Habazettl H, Kukucka M. A transapicalor transluminal approach to aortic valve implantation does not attenuate the inflammatory response. Heart Surg Forum. 2011 Apr;14(2):E110-3. PMID: 21521673.

2. Mota AL, Rodrigues AJ, Evora PR. Adult cardiopulmonary bypass in the twentieth century: science, art or empiricism?. Rev Bras Cir Cardiovasc. 2008 Jan-Mar;23(1):78-92. PMID: 18719832.

3. Warren OJ, Watret AL, de Wit KL, Alexiou C, Vincent C, Darzi AW, Athanasiou T. The inflammatory response to cardiopulmonary bypass: part 2--anti-inflammatory therapeutic strategies. J Cardiothorac Vasc Anesth. 2009 Jun;23(3):384-93. PMID: 19054695.

4. Warren OJ, Smith AJ, Alexiou C, Rogers PL, Jawad N, Vincent C, Darzi AW, Athanasiou T. The inflammatory response to cardiopulmonary bypass: part 1--mechanisms of pathogenesis. J Cardiothorac Vasc Anesth. 2009 Apr;23(2):223-31. PMID: 18930659.

5. Landis C. Pharmacologic strategies for combating the inflammatory response. J Extra Corpor Technol. 2007 Dec;39(4):291-5. PMID: 18293823.

6. Raja SG, Dreyfus GD. Modulation of systemic inflammatory response after cardiac surgery. Asian Cardiovasc Thorac Ann. 2005 Dec;13(4):382-95. PMID: 16304234.

7. Li YP, Huang J, Huang SG, Xu YG, Xu YY, Liao JY, Feng X, Zhang $\mathrm{XG}$, Wang JH, Wang J. The compromised inflammatory response to bacterial components after pediatric cardiac surgery is associated with cardiopulmonary bypass-suppressed Toll-like receptor signal transduction pathways. J Crit Care. 2014 Apr;29(2):312.e7-13. PMID: 24287173.

8. Durandy Y. Minimizing systemic inflammation during cardiopulmonary bypass in the pediatric population. Artif Organs. 2014 Jan;38(1):11-8. PMID: 24392866.

9. Allan CK, Newburger JW, McGrath E, Elder J, Psoinos C, Laussen PC, del Nido PJ, Wypij D, McGowan FX Jr. The relationship between inflammatory activation and clinical outcome after infant cardiopulmonary bypass. Anesth Analg. 2010 Nov;111(5):1244-51. PMID: 20829561

10. Hövels-Gürich HH, Vazquez-Jimenez JF, Silvestri A, Schumacher $\mathrm{K}$, Minkenberg R, Duchateau J, Messmer BJ, von Bernuth G, Seghaye MC. Production of proinflammatory cytokines and myocardial dysfunction after arterial switch operation in neonates with transposition of the great arteries. J Thorac Cardiovasc Surg. 2002 Oct;124(4):811-20. PMID: 12324741.

11. Onorati F, Rubino AS, Cuda A, Foti D, Sica V, Santini F, Gulletta E, Renzulli A. Impact of endothelial activation on infective and inflammatory complications after cardiac surgery in type II diabetes mellitus. Int J Artif Organs. 2011 Jun;34(6):469-80. PMID: 21574159.

12. Asimakopoulos G. Systemic inflammation and cardiac surgery: an update. Perfusion. 2001 Sep;16(5):353-60. PMID: 11565890.

13. Viaro F, Baldo CF, Capellini VK, Celotto AC, Bassetto S, Rodrigues AJ, Evora PR. Plasma nitrate/nitrite (NOx) is not a useful biomarker to predict inherent cardiopulmonary bypass inflammatory response. J Card Surg. 2008 Jul-Aug;23(4):336-8. PMID:18598323.

14. Davidson J, Tong S, Hauck A, Lawson DS, Jaggers J, Kaufman J, da Cruz E. Alkaline phosphatase activity after cardiothoracic surgery in infants and correlation with post-operative support and inflammation: a prospective cohort study. Crit Care. 2012 Aug 20;16(4):R160. PMID: 22906145.

15. Voisine P, Ruel M, Khan TA, Bianchi C, Xu SH, Kohane I, Libermann TA, Otu H, Saltiel AR, Sellke FW. Differences in gene expression profiles of diabetic and nondiabetic patients undergoing cardiopulmonary bypass and cardioplegic arrest. Circulation. 2004 Sep 14;110(1):II280-6. PMID: 15364876.

16. Wan S, LeClerc JL, Vincent JL. Inflammatory response to cardiopulmonar bypass: mechanisms involved and possible therapeutic strategies. Chest. 1997 Sep;112(3):676-92. PMID: 9315800 .

17. Gomes WJ, Carvalho AC, Palma JH, Gonçalves I Jr, Buffolo E. Vasoplegic syndrome: a new dilemma. J Thorac Cardiovasc Surg. 1994 Mar;107(3):942-3. PMID: 8127127.

18. Evora PR, Ribeiro PJ, de Andrade JC. Methylene blue administration in SIRS after cardiac operations. Ann ThoracSurg. 1997 Apr;63(4):1212-3. PMID:9124951.

19. Leite EG, Ronald A, Rodrigues AJ, Evora PR. Is methylene blue of benefit in treating adult patients who develop catecholamineresistant vasoplegic syndrome during cardiac surgery? Interact Cardiovasc Thorac Surg. 2006 Dec;5(6):774-8. PMID: 17670710.

20. Leyh RG, Kofidis T, Strüber M, Fischer S, Knobloch K, Wachsmann B, HaglC, Simon AR, Haverich A. Methylene blue: the drug of choice for catecholamine-refractory vasoplegia after cardiopulmonary bypass? J Thorac Cardiovasc Surg. 2003 Jun;125(6):1426-31. PMID: 12830064.

21. Levin RL, Degrange MA, Bruno GF, Del Mazo CD, Taborda DJ, Griotti JJ, Boullon FJ. Methylene blue reduces mortality and morbidity in vasoplegic patients aftercardiac surgery. Ann Thorac Surg. 2004 Feb;77(2):496-9. PMID: 14759425.

22. Ozal E, Kuralay E, Yildirim V, Kilic S, Bolcal C, Kücükarslan N, Günay C, Demirkilic U, Tatar H. Preoperative methylene blue administration in patients at high risk for vasoplegic syndrome during cardiac surgery. Ann Thorac Surg. 2005May;79(5):1615-9. PMID: 15854942.

23. Evora PR, Rodrigues AJ. Methylene blue revised. J Thorac Cardiovasc Surg. 2006 Jan;131(1):250-1. PMID: 16399329.

24. Evora PR, Ribeiro PJ, Vicente WV, Reis CL, Rodrigues AJ, Menardi AC, Alves Junior L, Evora PM, Bassetto S. Methylene blue for vasoplegic syndrome treatment in heart surgery: fifteen years of questions, answers, doubts and certainties. Rev Bras Cir Cardiovasc. 2009 Jul-Sep;24(3):279-88. PubMed PMID: 20011872.

25. Evora PR, Alves Junior L, Ferreira CA, Menardi AC, Bassetto S, Rodrigues AJ, ScorzoniFilho A, Vicente WV. Twenty years of vasoplegic syndrome treatment in heart surgery. Methylene blue revised. Rev Bras Cir Cardiovasc. 2015 Jan-Mar;30(1):84-92. PMID:25859872.

\section{Correspondence:}

Paulo Roberto Barbosa Evora

Rua Rui Barbosa, 367/15, Centro

14015-120 - Ribeirão Preto, São Paulo, Brasil

Tel./Fax: (55 16) 3602-2497

prbevora@fmrp.usp.br

Conflict of interest: none

Financial sources: Sao Paulo Research Foundation (FAPESP), National Council of Scientific and Technological Development (CNPq), Coordination of Improvement of Higher Academic Staff (CAPES).

${ }^{1}$ Research performed at Laboratory of Cardiovascular and Endothelium Function, Ribeirao Preto Medical School, University of Sao Paulo (FMRP-USP), Ribeirao Preto-SP, Brazil. Part of PhD degree thesis, Experimental Medicine Program. Tutor: Paulo Roberto Barbosa Evora. 